Research Paper

\title{
Prolidase, Matrix Metalloproteinases I and I 3 Activity, Oxidative-Antioxidative Status as a Marker of Preterm Premature Rupture of Membranes and Chorioamni- onitis in Maternal Vaginal Washing Fluids
}

Hatice Ender Soydinc ${ }^{\circledR}$, Muhammet Erdal Sak¹, Osman Evliyaoglu², Mehmet Siddık Evsen¹, Abdulkadir Turgut $^{1}$, Ali Özler ${ }^{1}$, İsmail Yıldız ${ }^{3}$, Talip Gul ${ }^{1}$

1. Department of Gynecology and Obstetrics, Faculty of Medicine, Dicle University, Diyarbakir, Turkey

2. Department of Biochemistry, Faculty of Medicine, Dicle University, Diyarbakir, Turkey

3. Department of Bioistatistic, Faculty of Medicine, Dicle University, Diyarbakir, Turkey

$\triangle$ Corresponding author: Dr. Hatice Ender SOYDINC, 1. Department of Gynecology and Obstetrics, Faculty of Medicine, Dicle University, Diyarbakir, Turkey. Email: endersoydinc@hotmail.com; Tel: +90 412 2488001/4904; Fax: +90412 2488523.

(c) Ivyspring International Publisher. This is an open-access article distributed under the terms of the Creative Commons License (http://creativecommons.org/ licenses/by-nc-nd/3.0/). Reproduction is permitted for personal, noncommercial use, provided that the article is in whole, unmodified, and properly cited.

Received: 2012.07.0I; Accepted: 2013.01.25; Published: 2013.08.15

\begin{abstract}
Objective: Etiology of premature preterm rupture of membranes (PPROM) is not yet completely known and chorioamnionitis is one of the most important complications of its. We aimed to evaluate whether prolidase, matrix metalloproteinases, oxidative-antioxidative status, and inflammation markers in vaginal washing fluid (VWF) were associated with etiology of PPROM and whether these markers could be used to predict chorioamnionitis in PPROM.
\end{abstract}

Study Design: This prospective case control study enrolled fifty pregnant women with PPROM and 50 healthy pregnant women. The VWF samples were taken at the time of admission in the PPROM group and patients were followed for chorioamnionitis. Prolidase, matrix metalloproteinases, oxidative-antioxidative status, and inflammation markers in VWF were assayed.

Results: VWF levels of prolidase, matrix metalloproteinases I-I3 $(p<0.00 I)$, oxidative stress parameters, total oxidative stress (TOS) $(p<0.001)$ and oxidative stress index (OSI) $(p=0.002)$, and hs-CRP ( $P=0.045)$ were significantly higher in the PPROM group than in the controls. Antioxidative status parameters, levels of paroxanase $(\mathrm{PON}-\mathrm{I})(\mathrm{p}<0.00 \mathrm{I})$ and total antioxidant capacity (TAC) $(p<0.001)$, were significantly lower in the PPROM group than in the controls. Mean VWF levels of prolidase $(p<0.001)$, metalloproteinases $(p<0.05)$, and oxidative-antioxidative status parameters $(p<0.05)$ were significantly different in women with versus women without chorioamnionitis in the PPROM group. Prolidase, MMP-I3, TOS, TAC, and PON-I were found as important predictors for chorioamnionitis in the PPROM group by the multivariate logistic regression analysis. When the ROC curve analysis for prolidase, MMP-I3, TOS, TAC, and PON-I were performed, all of them were statistically significant for area under the curve (areas under the curve were $0.94,0.90,0.80,0.25$, and 0.19 , respectively).

Conclusions: This study showed that collagen turnover mediators, especially prolidase, and increased oxidative stress are significantly associated with PPROM. Also, chorioamnionitis can be predicted with prolidase, MMP-I3, TOS, TAC, and PON-I in PPROM patients.

Key words: premature preterm rupture of membrane, chorioamnionitis, prolidase, matrix metalloproteinases, total oxidative status, total antioxidative capacity, paraoxonase-1, vaginal washing fluid. 


\section{Introduction}

Premature rupture of membranes (PROM) is described as the rupture of fetal membranes (amniorrhexis) prior to labor. If this occurs before the $37 \mathrm{th}$ week of gestation, it is called preterm premature rupture of membranes (PPROM). PPROM occurs in approximately $3 \%$ of all pregnancies and it comprises one-third of all premature deliveries $(1,2)$. It is considered a major cause of perinatal morbidity and mortality in the world. The etiology of PPROM includes maternal age, low maternal body mass index (BMI), smoking, genital tract infections, excessive membrane stretch (uterine overdistension), and placental abruption. The pathophysiology of PPROM is not clear, but disorders of collagen metabolism, increased oxidative stress, and choriodecidual inflammation and infection have been addressed (1,3-5).

Matrix metalloproteinases (MMPs) are enzymes that cause extracellular matrix degradation. The MMP family consists of 25 different enzymes, and a number of those have been detected in fetal membranes (6-8). Tissue support of the fetal membranes is generated by type I and type III collagens, which are the predominant collagens of the chorioamnion extracellular matrix. Chorioamnion extracellular matrix collagen degradation is regulated by MMPs. Although the mechanism of extracellular matrix degradation has not been fully understood, several studies have shown evidence that MMPs may play a role in PPROM $(9,24)$. Prolidase, a member of the MMP family, is a cytosolic enzyme that participates in the regulation of collagen biosynthesis $(10,11)$. It hydrolyzes imidodipeptides and imidotripeptides with C-terminal proline or hydroxyproline. Because of its high levels of imino acids, collagen is an important substrate of prolidase. A relation between increased prolidase activity and diseases involving tissue destruction such as cancer, tuberculosis, and chronic liver disease has been found (29-31). Also, increased prolidase activity in the placental tissue has been shown to be associated with early pregnancy loss (12). All of these studies suggest that an increase in prolidase activity is correlated with increased rates of collagen turnover and dysregulation of collagen metabolism.

Oxidative stress occurs when reactive oxygen substances (ROS) fail to be scavenged after metabolic processes. ROS-induced oxidative damage can cause increased cell membrane permeability, increased susceptibility of proteins to proteolysis, and DNA damage. To protect cells from the deleterious effect of ROS, antioxidants rapidly and efficiently remove ROS from the intracellular environment. Catalase, glutathione peroxidase, and paraoxonase are some of the enzymatic antioxidants. Catalase contributes to ROS detoxification by converting $\mathrm{H}_{2} \mathrm{O}_{2}$ to water, glutathione peroxidase by metabolizing hydrogen peroxide and lipid hydroperoxides, and paraoxonase by hydrolyzing organophosphoruses and toxic oxidized lipids. The ratio of TOS to TAS levels, known as oxidative stress index (OSI), is an indicator of the degree of oxidative stress (18). Increased ROS may alter cell biology and may induce collagen catabolism. In vitro ROS exposure of amniocytes was found to result in an increase in $\mathrm{Ca}^{2}$ and $\mathrm{pH}$, a decrease in $\mathrm{Mg}^{2}$, and the release of arachidonic acid, similar to the intracellular changes that are seen in PPROM (13). Another study reported that when cultured chorioamnion segments were exposed to superoxide, MMP-9 activity was increased (32).

Patients with PPROM who also have chorioamnionitis have been found to have increased maternal and fetal complications. Maternal complications include endomyometritis, wound infection, pelvic abscess, bacteremia, and postpartum hemorrhage, while fetal complications include fetal death, neonatal sepsis, and fetal inflammatory response syndrome. For these reasons, the early diagnosis of chorioamnionitis in patients with PPROM is crucial. Many studies have evaluated the efficacy of specific biomarkers for intraamniotic infection with noninvasive methods, and in different studies IL-6, monocyte chemotactic protein- 1 , and tumor necrosis factor were found to be related with intraamniotic infection and histologic chorioamnionitis $(14,15)$.

Increased metalloproteinase activity is thought to take place in the pathologic cascade of PPROM. Different members of the MMP family have been shown to be related with PPROM, but to the best of our knowledge, there is no study in the literature that evaluates the relation between PPROM and prolidase activity. Because of its high fetal and maternal complication risk, early detection of chorioamnionitis is very important for carrying out optimal management in patients with PPROM. In this study we aimed to determine (i) whether matrix metalloproteinase, prolidase, and oxidative stress markers are related to PPROM, and (ii) whether these markers could be used to predict chorioamnionitis in PPROM.

\section{Materials and Methods}

\section{Patients}

This prospective case-controlled study was conducted at the Department of Obstetrics and Gynecology at Dicle University Medical Hospital from January 2011 to December 2011. Patients who were consecutively admitted to our clinic with a diagnosis of PPROM and subjects with healthy pregnancies who 
came for routine prenatal visits were recruited to our study. The protocol was approved by the Ethics Committee of Dicle University. All subjects provided informed consent.

We included 100 pregnant women that were at 26-34 gestational weeks. Fifty of these patients had a diagnosis of PPROM (PPROM group) and 50 of them had healthy pregnancies (control group). Patients with preeclampsia, placenta previa, abruptio placenta, polyhydramnios, multiple pregnancy, major fetal anomaly, diabetes mellitus, chronic systemic disease, and doubtful PPROM diagnosis were excluded from the study. Rupture of membranes was diagnosed with a combination of vaginal pooling of amniotic fluid and a positive nitrazine test by an examination with a sterile speculum. As a standard protocol of our institution, biochemical tests (glucose, aspartate transaminase, alanine transaminase, lactate dehydrogenase, creatinine, blood urea nitrogen) and complete blood count (CBC) were performed to evaluate patients' systemic disorders, and a urinalysis was performed to exclude urinary infection. An obstetric ultrasound (USG) was also performed to determine gestational age, define any major anomalies, and determine the amniotic fluid index and number of fetuses.

All of the women with PPROM were hospitalized in our obstetric clinic. Expectant management was implemented for all pregnant women with PPROM except for those having complications such as chorioamnionitis, nonreassuring fetal testing, and active labor. All of the women were given prophylactic antibiotic therapy (with ampicillin) as medical intervention. Also, standard antenatal corticosteroid therapy (with betamethasone) was administered for accelerating fetal lung maturation in pregnant women with PPROM before 32 weeks of gestation (1). Patients were delivered if (or when) fetal distress, active labor, or clinical chorioamnionitis developed.

Patients were closely followed for possible clinical infections. Patients with clinical chorioamnionitis were described as having two or more of the following criteria: presence of uterine tenderness and irritability, foul smelling and purulent discharge, leukocytosis $\left(>15.000 / \mathrm{mm}^{3}\right)$, and/or maternal fever $(>38$ $\left.{ }^{\circ} \mathrm{C}\right)$. Antibiotic prophylaxis was administered to patients in the PPROM group according to routine treatment protocol, while corticosteroid therapy was given to pregnant women earlier than 32 gestational weeks in the PPROM group.

The histopathological examination of the placenta was evaluated with standard published protocols (16). Histologic chorioamnionitis was defined as the presence of acute inflammatory changes upon examination of a membrane roll and chorionic plate of the placenta; funisitis was diagnosed as the visualiza- tion of neutrophil infiltration into the umbilical vessel walls or Wharton's jelly.

If patients with PPROM were complicated with clinical and/or histopathologic chorioamnionitis, they were defined as chorioamnionitis (+); otherwise, they were defined as chorioamnionitis (-).

Cases in the control group were selected from patients who were at 26-34 gestational weeks, had no complications during routine prenatal visits, and delivered at term.

\section{Sampling}

The vaginal washing fluid (VWF) sampling was done as follows. First, a sterile speculum examination was performed on all patients, where $5 \mathrm{ml}$ of sterile saline solution was injected into the vagina. With the same syringe, the vaginal washing fluid was aspirated from the posterior vaginal fornix. The fluid sample was immediately centrifuged at $3000 \mathrm{rpm}$ for $10 \mathrm{~min}$ and the supernatant was stored at $-80^{\circ} \mathrm{C}$ until analysis.

The VWF samples were taken at the time of admission in the PPROM group, while they were taken during routine prenatal visits for control group.

\section{Biochemical Analysis of Parameters}

\section{Measurement metalloproteinases and prolidase activity}

Prolidase activity was determined by a spectrophotometric method, which is devoted to the measurement of the proline levels produced by prolidase. After the supernatant was diluted with physiological saline, the other processes were performed as mentioned in previous studies (17).

Specific and sensitive commercially available enzyme-linked immunosorbent assays (ELISA) (Raybiotech Inc., USA) were used to determine concentrations of MMP-1 and MMP-13 levels in vaginal washing fluid.

\section{Measurement of total oxidant-antioxidant status}

Total oxidative status (TOS) and total antioxidant status (TAS) were measured by an automated method as mentioned in previous studies (17). In this method, measuring TOS is based on spectrophotometrically measuring color intensity of ferric ions, generated by oxidation of $\mathrm{Fe}^{2}$ to $\mathrm{Fe}^{3}$, and measuring TAS is based on determining total antioxidant response against potent biological free hydroxyl radicals. Oxidative stress index [OSI (arbitrary unit) = TOS ( $\mu$ mol H2O2 equiv/L) / TAC (mmol Trolox equiv/L)] was calculated as the percent ratio of TOS to TAC. 
For the enzymatic antioxidants, catalase activity was measured according to the method of Aebi, and paraoxonase-1 (PON-1) activity was measured spectrophotometrically by a modified Eckerson method $(19,20)$. Another antioxidant enzyme glutathione peroxidase (GPx) activity was determined by the coupled assay procedure of Gunzler et al. (21). One unit of enzyme activity is reported as one $\mu \mathrm{mol}$ of NADPH oxidized per minute.

\section{Measurement inflammation markers}

An enzyme-linked immunosorbent assay (ELISA) (Dynex Technologies, Inc. Chantilly, VA, USA) was used for the analysis of tumor necrosis factor-alpha (TNF-a) and high sensitivity C-reactive protein (hs-CRP) levels (Diasource Immunoassays S.A., Belgium).

\section{Other parameters}

Complete blood count was measured using the Abbott Cell Dyn 3700 Hematology Analyzer (Abbott Laboratories, Abbott Park, IL, USA). Serum biochemical parameters were assayed using an autoanalyzer Architect C 16000 (Abbott Laboratories, Abbott Park, IL, USA), and urine analysis was performed using the IQ 200 urine analyzer (Iris Diagnostics, Chatsworth, CA, USA).

\section{Statistical methods}

Data were analyzed using the Microsoft Statistical Package for Social Sciences (SPSS) version 18.0 for Windows. The sample size was calculated by web site of Daniel Soper Statistics Calculators using anticipated effect size (Cohen's $d$ ) $=0.8,80 \%$ power, alpha $=0.05$, and considering the results of previous studies (22).

The Kolmogorov-Smirnov test was used to determine normal distribution of data. According to normal and abnormal distribution, unpaired comparisons of continuous variables were performed with a Mann-Whitney U or Student's t-test, and categorical studied variables were compared by Chi-Square, Pearson Chi-Square, or Fisher's Exact Tests. Logistic regression analyses were performed to detect whether statistically significant parameters related to PPROM can also predict PPROM. Logistic regression analyses were also performed to detect whether parameters related to chorioamnionitis can predict infection in patients with PPROM. For determining the sensitivity, specificity, and positive and negative predictive values of the parameters, receiver-operating characteristics (ROC) curve analysis was performed. $P$-values $<0.05$ were considered significant.

\section{Results}

Fifty women with PPROM delivering at $<34$ weeks constituted the PPROM group, five of whom $(10 \%)$ had a history of PPROM. Fifty uncomplicated pregnancies delivering at term constituted the control group, three of whom $(6 \%)$ had a history of PPROM. There were no differences in demographic parameters between women in the PPROM and control groups (Table 1).

Vaginal washing fluid (VWF) levels of prolidase ( $\mathrm{p}<0.001)$, MMP-1 ( $<0.001)$, MMP-13 (p < 0.001), TOS ( $\mathrm{p}<0.001)$, OSI $(\mathrm{p}=0.002)$, and hs-CRP $(\mathrm{p}=$ 0.045 ) were significantly higher in the PPROM group than in the control group. VWF levels of PON-1 ( $p<$ $0.001)$, TAC $(p=0.010)$, and CAT $(p=0.035)$ were significantly lower in the PPROM group than in the control group. There were no statistically significant differences in GPx and TNF-a levels between the two groups (Table 2).

Table I: Demographic characteristics

\begin{tabular}{llll}
\hline & PPROM group & $\begin{array}{l}\text { Controls } \\
\text { group }\end{array}$ & P value \\
\hline Maternal age (years) & $28.9 \pm 4.80$ & $29.2 \pm 6.80$ & 0.852 \\
\hline Parity & $2.18 \pm 1.99$ & $2.120 \pm 1.95$ & 0.850 \\
\hline Abortus & $0.65 \pm 1.29$ & $0.37 \pm 0.89$ & 0.254 \\
\hline $\begin{array}{l}\text { Gestational age at } \\
\text { assessment (weeks) }\end{array}$ & $30.75 \pm 3.89$ & $31.10 \pm 3.94$ & 0.684 \\
\hline History of PPROM & $5(10)$ & $3(6)$ & 0.715 \\
\hline BMI (kg/m $)$ & $27.5 \pm 1.68$ & $27.8 \pm 1.65$ & 0.527 \\
\hline
\end{tabular}

Values are given as mean \pm SD or $\mathrm{n}(\%)$. BMI, body mass index.

Table 2: Vaginal washing fluid levels of parameters in women with PPROM and in control women

\begin{tabular}{llll}
\hline & $\begin{array}{l}\text { PPROM } \\
\text { group } \\
\text { (N:50) }\end{array}$ & $\begin{array}{l}\text { Controls } \\
\text { group } \\
\text { (N:50) }\end{array}$ & P value \\
\hline Prolidase (U/L) & $912.1 \pm 89.6$ & $592.3 \pm 69.8$ & $<0.001$ \\
MMP 1 (pg/ml) & $144.1 \pm 25.20$ & $96.95 \pm 16.64$ & $<0.001$ \\
MMP 13 (pg/ml) & $63.14 \pm 15.40$ & $38.42 \pm 6.14$ & $<0.001$ \\
TOS ( $\mathbf{p m o l ~ H 2 O 2 ~ E q u i v . / L ) ~}$ & $9.96 \pm 1.80$ & $8.39 \pm 1.16$ & $<\mathbf{0 . 0 0 1}$ \\
TAC (mmolTrolox Equiv./L) & $1.44 \pm 0.33$ & $1.80 \pm 0.43$ & $\mathbf{0 . 0 1 0}$ \\
GPx (U/ml) & $1.21 \pm 0.33$ & $1.41 \pm 0.35$ & 0.103 \\
CAT (U/L) & $587.3 \pm 53.6$ & $657.4 \pm 46.3$ & $\mathbf{0 . 0 3 5}$ \\
PON 1 (U/L) & $42.82 \pm 8.61$ & $54.97 \pm 9.95$ & $<0.001$ \\
TNF- a (pg/ml) & $19.13 \pm 5.90$ & $17.66 \pm 5.23$ & 0.57 \\
hs-CRP (mg/dl) & $0.90 \pm 0.32$ & $0.62 \pm 0.24$ & $\mathbf{0 . 0 4 5}$ \\
OSI (Arbitrary Unite) & $8.05 \pm 2.25$ & $5.27 \pm 1.85$ & $\mathbf{0 . 0 0 2}$ \\
\hline
\end{tabular}

Values are reported as mean \pm standard deviation (SD). MMP 1: matrix metalloproteinase 1; MMP 13: matrix metalloproteinase 13; TOS: total oxidative status; TAC: total antioxidant capacity; GPx: glutathion peroxidase; CAT: catalase; PON 1: paraoxonase 1; TNF-a: tumor necrosis factor alpha; hs-CRP: high sensitivity C-reactive protein; OSI: oxidative stres index. 
A multivariate logistic regression analysis was performed for statistically significant parameters in VWF. Prolidase, MMP-1, MMP-13, TOS, TAC, and PON-1 were all found to be important predictors for PPROM in VWF (Table 3).

Clinical chorioamnionitis was diagnosed in 7 women in the PPROM group (14\%). Of the clinical chorioamnionitis parameters, leukocytosis was observed in 12 patients (24\%), fever was observed in 7 patients $(14 \%)$, foul-smelling discharge was observed in 7 patients $(14 \%)$, and uterine sensitivity was observed in 3 patients $(6 \%)$. Histologic evidence of chorioamnionitis was found in 13 women $(26 \%)$ in the PPROM group during placental examinations.

Patients with and without chorioamnionitis in the PPROM group were compared with respect to biochemical markers. We found that prolidase, MMP-1, MMP-13, TOS, and OSI were significantly higher in those with chorioamnionitis, while TAC and PON-1 were found to be significantly lower in patients with chorioamnionitis (Table 4). After performing a multivariate logistic regression analysis for these markers, we found that prolidase, MMP-13, TOS, TAC, and PON-1 were important predictors for chorioamnionitis (Table 5). Next, a ROC curve analysis was performed for intrauterine infection predictors (Figure 1). In the ROC curve analysis, the optimal cut-off values for the detection of chorioamnionitis were $>1052$ for prolidase with AUC equal to 0.94 (SE $=$ $0.02, \mathrm{p}=0.001) ;>56$ for MMP-13 with AUC equal to $0.90(\mathrm{SE}=0.03, \mathrm{p}=0.001) ;>10.5$ for TOS with AUC equal to 0.80 (SE $=0.05, \mathrm{p}=0.001) ; \leq 1.18$ for TAC with AUC equal to $0.25(\mathrm{SE}=0.07, \mathrm{p}=0.003)$; and $\leq 41.41$ for PON-1 with AUC equal to $0.19(\mathrm{SE}=0.02, \mathrm{p}=0.001)$. The diagnostic indices for the aforementioned parameters' cut-offs are presented in Table 6.

Table 3: The multivariate logistic regression analysis of parameters in women with PPROM

\begin{tabular}{llll}
\hline & Beta-coefficient & P value & Odds ratio $(\mathbf{9 5} \%$ CI) \\
\hline Prolidase & -0.012 & $\mathbf{0 . 0 0 2}$ & $0.988(0.980-0.995)$ \\
MMP 1 & -0.029 & $\mathbf{0 . 0 2 1}$ & $0.972(0.948-0.996)$ \\
MMP 13 & -0.152 & $\mathbf{0 . 0 1 4}$ & $0.859(0.760-0.970)$ \\
TOS & -0.643 & $\mathbf{0 . 0 0 4}$ & $0.526(0.337-0.819)$ \\
TAC & 1.631 & $\mathbf{0 . 0 0 7}$ & $5.107(1.565-5.668)$ \\
CAT & 0.001 & 0.977 & $1.000(0.995-1.005)$ \\
PON 1 & 0.144 & $\mathbf{0 . 0 0 1}$ & $1.155(1.065-1.252)$ \\
hs-CRP & -1.537 & 0.157 & $0.215(0.026-1.807)$ \\
OSI & -0.040 & 0.893 & $0.960(0.532-1.733)$ \\
\hline
\end{tabular}

CI: confidence interval. MMP 1: matrix metalloproteinase 1; MMP 13: matrix metalloproteinase 13; TOS: total oxidative status; TAC: total antioxidant capacity CAT: catalase; PON 1: paraoxonase 1; hs-CRP: high sensitivity C-reactive protein; OSI: oxidative stres index.
Table 4: Vaginal washing fluid levels of parameters in women with and without chorioamnionitis in PPROM group

\begin{tabular}{|c|c|c|c|}
\hline Parameters & $\begin{array}{l}\text { Chorioamnionitis } \\
(+) \\
(\mathrm{N}: 13)\end{array}$ & $\begin{array}{l}\text { Chorioamnionitis } \\
(-) \\
(\mathrm{N}: 37)\end{array}$ & $\begin{array}{l}P \\
\text { value }\end{array}$ \\
\hline Prolidase (U/L) & $1090.3 \pm 78.11$ & $820.1 \pm 69.97$ & $<0.001$ \\
\hline MMP 1 (pg/ml) & $183.3 \pm 32,57$ & $123.6 \pm 22.55$ & 0.007 \\
\hline MMP 13 (pg/ml) & $77.58 \pm 15.05$ & $55.67 \pm 11.42$ & 0.003 \\
\hline $\begin{array}{l}\text { TOS }(\mu \mathrm{mol} \mathrm{H} 2 \mathrm{O} 2 \\
\text { Equiv./L) }\end{array}$ & $10.89 \pm 1.51$ & $9.48 \pm 1.78$ & 0.023 \\
\hline $\begin{array}{l}\text { TAC (mmolTrolox } \\
\text { Equiv./L) }\end{array}$ & $1.21 \pm 0.25$ & $1.56 \pm 0.17$ & 0.015 \\
\hline $\mathrm{GPx}(\mathrm{U} / \mathrm{ml})$ & $1.13 \pm 0.30$ & $1.26 \pm 0.39$ & 0.385 \\
\hline CAT (U/L) & $539.2 \pm 79,8$ & $612.2 \pm 84,9$ & 0.077 \\
\hline PON 1 (U/L) & $38.10 \pm 8.26$ & $45.26 \pm 9.11$ & 0.044 \\
\hline TNF- $\alpha(p g / m l)$ & $21.96 \pm 5.99$ & $17.67 \pm 4.38$ & 0.407 \\
\hline hs-CRP (mg/dl) & $0.79 \pm 0.34$ & $0.95 \pm 0.47$ & 0.833 \\
\hline OSI (Arbitrary Unite) & $11.0 \pm 2.72$ & $6.52 \pm 1.33$ & 0.004 \\
\hline
\end{tabular}

Values are reported as mean \pm standard deviation (SD). MMP 1: matrix metalloproteinase 1; MMP 13: matrix metalloproteinase 13; TOS: total oxidative status; TAC: total antioxidant capacity; GPx: glutathion peroxidase; CAT: catalase PON 1: paraoxonase 1; TNF-a: tumor necrosis factor alpha; hs-CRP: high sensitivity C-reactive protein; OSI: oxidative stres index.

Table 5: The multivariate logistic regression analysis of parameters in women with chorioamnionitis in PPROM group

\begin{tabular}{llll}
\hline & Beta-coefficient & P value & Odds ratio $(\mathbf{9 5} \%$ CI) \\
\hline Prolidase & 0.016 & $\mathbf{0 . 0 0 7}$ & $1.016(1.004-1.027)$ \\
MMP 1 & 0.830 & 0.991 & $2.294(0.948-1.860)$ \\
MMP 13 & 0.093 & $\mathbf{0 . 0 3 0}$ & $1.098(1.009-1.194)$ \\
TOS & 0.730 & $\mathbf{0 . 0 5}$ & $2.074(0.748-5.749)$ \\
TAC & -5.248 & $\mathbf{0 . 0 2 8}$ & $0.005(0.001-0.563)$ \\
PON 1 & -0.111 & $\mathbf{0 . 0 1 3}$ & $0.895(0.819-0.977)$ \\
OSI & -12.669 & 0.996 & $0.001(0.532-1.733)$
\end{tabular}

CI: confidence interval. MMP 1: matrix metalloproteinase 1; MMP 13: matrix metalloproteinase 13; TOS: total oxidative status; TAC: total antioxidant capacity; PON 1: paraoxanase 1; OSI: oxidative stres index.

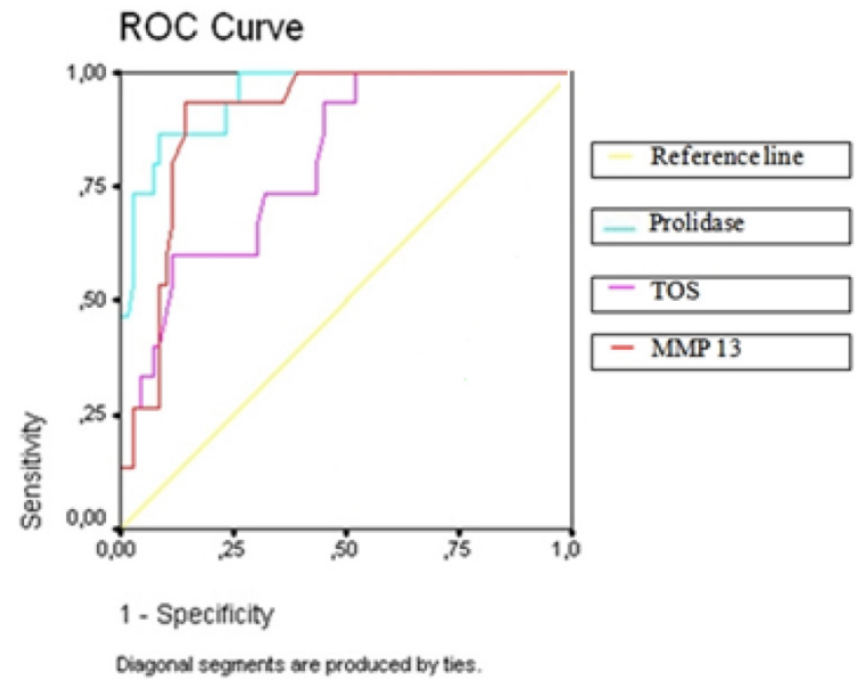

Figure I: The Receiver Operating Characteristic (ROC) curve analysis of significant parameters in women with chorioamnionitis in PPROM group (TOS: total oxidative status; MMP 13: matrix metalloproteinase 13) 
Table 6: Sensitivity, specificity, positive (PPV) and negative (NPV) predictive values and likelihood ratios (LR) for the optimal cut-offs value of the parameters for chorioamnionitis predicted

\begin{tabular}{lllllll}
\hline Variables & $\begin{array}{l}\text { Sensitivity }(\%) \\
(95 \% \mathrm{CI})\end{array}$ & $\begin{array}{l}\text { Specificity(\%) } \\
(95 \% \mathrm{CI})\end{array}$ & $\begin{array}{l}\mathrm{PPV}(\%) \\
(95 \% \mathrm{CI})\end{array}$ & $\begin{array}{l}\text { NPV(\%) } \\
(95 \% \mathrm{CI})\end{array}$ & $\begin{array}{l}\text { LR+ } \\
(95 \% \mathrm{CI})\end{array}$ & $\begin{array}{l}\mathrm{LR}- \\
(95 \% \mathrm{CI})\end{array}$ \\
\hline Prolidase $>\mathbf{1 0 5 2 . 7}$ & 73.33 & 93.10 & 84.6 & 87.1 & 10.63 & 0.29 \\
& $(44.9-92.0)$ & $(77.2-99.0)$ & $(54.5-97.6)$ & $(70.1-96.3)$ & $(7.7-14.7)$ & $(0.06-1.4)$ \\
MMP13 $>$ 56 & 93.33 & 65.52 & 58.3 & 95 & 2.71 & 0.10 \\
& $(68.0-98.9)$ & $(45.7-82.0)$ & $(36.7-77.9)$ & $(75.1-99.2)$ & $(2.0-3.6)$ & $(0.01-0.7)$ \\
TOS $>\mathbf{1 0 . 5}$ & 60.0 & 72.41 & 52.9 & 77.8 & 2.17 & 0.55 \\
& $(32.3-83.6)$ & $(52.8-87.2)$ & $(27.9-77.0)$ & $(57.7-91.3)$ & $(1.4-3.5)$ & $(0.2-1.3)$ \\
\hline
\end{tabular}

CI: confidence interval. MMP 13: matrix metalloproteinase 13; TOS: total oxidative status

\section{Discussion}

PPROM is still a matter of debate in perinatology regarding its unclear etiology and controversies over follow-up and management of patients with a PPROM diagnosis. Disorders of collagen metabolism, increased oxidative stress, and choriodecidual inflammation and infection are thought to be etiologic factors. In our study we found that patients with PPROM had high prolidase, MMP-1, MMP-13, TOS, OSI, and hCRP levels and low TAC, CAT, and PON-1 levels in their VWF. Logistic regression analysis showed that prolidase, MMP-1, MMP-13, TOS, TAC, and PON-1 in VWF were associated with PPROM. These finding showed for the first time that PPROM may be a result of collagen damage caused by increased prolidase activity. On the other hand, positive association between PPROM and increased metalloproteinase activity and oxidative stress in our study is comparable to the literature. In women who developed chorioamnionitis during the clinic follow-up in the PPROM group, prolidase, MMP-1, MMP-13, TOS, and OSI were significantly higher, and TAC and PON-1 were found to be significantly lower than in women without chorioamnionitis.

The degradation of collagen in the chorioamnion, which is composed of intensive connective tissue, is regulated by matrix metalloproteinases. MMP-1, known as collagenase I, and MMP-13, known as collagenase III, are two members of this family, and they are found in fetal membranes and amniotic fluid (23). MMP-1 is secreted by amniotic mesenchymal cells and degrades type I, II, and III collagens. MMP-13 is a member of the interstitial collagenase family and has collagenolytic and gelatinolytic activity. Many studies reported that patients with PPROM have increased MMP activation. Biggio et al. reported that "women with midtrimester MMP-8 levels greater than the 90th percentile were over 3 times more likely to experience PPROM than women with lower levels" (24). Maymon et al. reported that MMP-7 levels were increased in patients with intraamniotic infection, which may facilitate extracellular matrix destruction (25). Athayde et al. found a significant increase in MMP-9 levels in amniotic fluids of patients with PPROM, and proposed that MMP-9 might be responsible for PROM (26). We found that MMP-1 and MMP-13 levels were higher in the PPROM group than the controls. MMP-1 and MMP-13 levels were also found to be higher in VWFs of PPROM patients with chorioamnionitis than without chorioamnionitis. Maymon et al. showed that preterm premature rupture of membranes -in both the presence and absence of infection - was associated with an increase in MMP-1 concentrations in the amniotic fluid (27). They concluded that MMP-1 is implicated in the mechanism of membrane rupture. MMP-1,7,8 and 9 were found to be increased in PROM in previous studies; however, studies for MMP-13 are relatively few. Fortunato et al. failed to determine any association between MMP-13 and PROM (23). They found that the concentration of MMP-13 is lower in PPROM than in the pre-term labor group, and they concluded that MMP-13 may not play a significant role in PROM. In contrast, we found that MMP-13 was associated with PPROM in our study. The difference between Fortunato's and our study might be attributable to study population, because in our study we included PPROM and healthy pregnant women with similar gestational age but as mentioned above Fortunato compared pregnant women with PPROM and pre-term labor. In patient with pre-term labor MMP-13 levels can be different from healthy pregnant women.

One important finding in our study was that prolidase levels were significantly higher in the PPROM group. To the best of our knowledge, this is the first study that detected high levels of prolidase in patients with PPROM. Prolidase facilitates matrix remodeling and cell infiltration and regulates cytokines and other immune modulators, and this proteolytic enzyme is one of the members of the MMP family that mediates the last step of collagen degradation. Its activity has been documented in erythrocytes, leukocytes, plasma, dermal fibroblasts, the 
kidney, brain, heart, thymus, and uterus (28). It has been proposed that there is an association between prolidase activity and diseases that are characterized by collagen turnover. Increased prolidase activity and increased extracellular matrix destruction have been detected in chronic hepatic disorders, cancers, and tuberculosis (29-31). When we take into account the increased levels of MMP-1 MMP-13 and prolidase in the PPROM group, we believe that the excessive degradation of the extracellular matrix of amniochorion plays an important role in PPROM etiology. Dynamic collagen metabolism is required for fetal membrane integrity during a normal pregnancy. MMP activity is responsible for collagen metabolism, and this activity is regulated by transcription and translation processes and also by specific regulators (tissue inhibitors of metalloproteinase, or TIMPs). Excessive MMP activity that is induced by inflammation, infection, obstetric reasons (multiple gestation, polyhydramnios, cervical pathologies, bleeding), smoking, and nutritional status may disturb the balance of collagen turnover (which is essential for fetal membranes) in favor of destruction.

MMPs have destructive effects on tissues and can be stimulated by reactive oxygen species (ROS). ROS may also activate genes that code MMPs and proinflammatory cytokines. It has been shown that collagenolytic enzymes in chorioamnion are sensitive to ROS stimulation (32). Increased MMP activity after ROS stimulation may cause collagen degradation and dissociation, which may lead to PPROM. Oxidative stress (OS) is defined as an imbalance in the production of reactive oxygen species and the capacity of antioxidant defenses. There are limited data in the literature about oxidative stress in PPROM. Longini et al. found that isoprostanes (F2-IP), which are direct indicators of oxidative stress, were increased in the amniotic fluid of patients with PROM (33). Osaikhuwuomwan et al. found decreased levels of vitamin $C$, which is an important antioxidant, in patients with PPROM (34). We used TOS as an oxidative marker, while CAT, PON-1, GPx, and TAC were used as antioxidant markers, and we investigated their effects on PPROM. TOS was increased, but TAC, PON-1, and CAT were decreased in the PPROM group when compared to the control group. OSI, which reflects the balance between oxidation and antioxidation, was signsignificantly higher in the PPROM group. Our findings suggest that PPROM may occur as a result of oxidative stress-induced damage at the chorioamnion epithelium or its collagen structure.

Immediate delivery of the baby is the preferred therapeutic approach in patients with PPROM who also have signs of intrauterine infection. Therefore, close follow-up of patients with PPROM with respect to intrauterine infection is crucial. The gold standard for the diagnosis of intrauterine infection is the isolation of microorganisms in amniocentesis samples. Although amniocentesis-related complications are seen in only $3.6 \%$ of patients in their third trimester, pain, discomfort, and anxiety are common with this procedure and therefore impose limitations on its daily practice (35). Given the risk of maternal and fetal complications of intrauterine infections, particularly neonatal white matter disease and cerebral palsy, non-invasive methods for the diagnosis of chorioamnionitis before symptomatic disease are increasingly important. In our study, prolidase, MMP-13, PON-1, TAC, and TOS were found to be important predictors of chorioamnionitis. Sensitivities of prolidase, MMP-13, and TAC were $73 \%, 93 \%$, and $60 \%$, respectively, whereas their specificities were $93 \%, 65 \%$, and $89 \%$, respectively. We would suggest to address that these findings are promising so that particularly prolidase may be used in VWF sampling as a non-invasive screening method for chorioamnionitis but external validation with randomized clinical trials is needed before.

Potential limitations of our study should be mentioned: Our study are relatively limited number of study subjects and cross-sectional study design. The cross-sectional design limited our ability to deduce association between PPROM and metalloproteinases, oxidative-antioxidative status, and inflammation markers. The samples were taken only at the time of admission in the PPROM group. The strength of predicting chorioamnionitis could be increased by samples were taken certain intervals. However, this is a first study evaluating especially prolidase activity in patient with PPROM and chorioamnionitis.

In conclusion, the MMPs that play a role in collagen metabolism and increased oxidative stress may indicate amniochorion rupture and also may be etiologic factors for PPROM. In this study, increased prolidase activity has been shown in PPROM patients for the first time, which may reflect that increased collagen turnover is a part of PPROM. Besides its role in the pathologic process of PPROM, prolidase has a high specificity in predicting chorioamnionitis, and therefore it may be used as a non-invasive test for the diagnosis. Further studies are required to investigate the benefits of prolidase as a diagnostic test to detect chorioamnionitis on improvements in maternal and fetal complications during follow-up or treatment of patients with PPROM.

\section{Acknowledgements}

This study was supported by Scientific Research and Project Coordinator (DUBAB, 10-TF-57) in Dicle 


\section{University.}

\section{Competing Interests}

The authors have declared that no competing interest exists.

\section{References}

1. Mercer BM. Preterm premature rupture of the membranes. Obstet Gynecol 2003; 101: 178-93.

2. Lawrence I. Obstetrics and Gynaecology. Massachusetts: Blackwell Publishing, 2004.

3. Parry S, Strauss III JF. Premature rupture of the fetal membranes. N Engl J Med 1998; 338: 663-70.

4. Mercer BM, Goldenberg RL, Meis PJ, et al. The Preterm Prediction Study: prediction of preterm premature rupture of membranes through clinical findings and ancillary testing. The National Institute of Child Health and Human Development Maternal- Fetal Medicine Units Network. Am J Obstet Gynecol 2000; 183: 738-45.

5. Woods JR Jr. Reactive oxygen species and preterm premature rupture of membranes-a review. Placenta 2001; 22 (Suppl A): 38-44.

6. Fortunato SJ, Menon R, Lombardi SJ. Collagenolytic enzymes (gelatinases) and their inhibitors in human amniochorionic membrane. Am J Obstet Gynecol 1997; 177: 731-41.

7. Fortunato SJ, Menon R, Lombardi SJ. Amniochorion gelatinase-gelatinase inhibitor imbalance in vitro: A possible infectious pathway to rupture. Obstet Gynecol 2000; 95: 240-44.

8. Fujimoto T, Parry S, Urbanek M, Sammel M, Macones G, Kuivaniemi H, Romero R, Strauss JF 3rd. A single nucleotide polymorphism in the matrix metalloproteinase-1 (MMP-1) promoter influences amnion cell MMP-1 expression and risk for preterm premature rupture of the fetal membranes. J Biol Chem. 2002; 277: 6296-302.

9. Menon R, Fortunato SJ. The role of matrix degrading enzymes and apoptosis in rupture of membranes. J Soc Gynecol Investig 2004; 11: 427-37.

10. Muszynska A, Pałka J, Gorodkiewicz E. The mechanism of daunorubicin induced inhibition of prolidase activity in human skin fibroblasts and its implication to impaired collagen biosynthesis. Exp Toxicol Pathol 2000; 52: $149-55$.

11. Emmerson KS, Phang JM. Hydrolysis of proline dipeptides completely fulfills the proline requirement in a proline-auxotropic Chinese hamster ovary cell line. J Nutr 1993; 123: 909-14.

12. Vural M, Toy H, Camuzcuoglu H, Aksoy N. Comparison of prolidase enzyme activities of maternal serum and placental tissue in patients with early pregnancy failure. Arch Gynecol Obstet 2011; 283: 953-8.

13. Ikebuchi Y, Masumoto N, Tasaka K, Koike K, Kasahara K, Miyake A, Tanizawa O. Superoxide anion increases intracellular $\mathrm{pH}$, intracellular free calcium, and arachidonate release in human amnion cells. J Biol Chem 1991; 266: 13233-7.

14. Jung HJ, Park KH, Kim SN, Hong JS, Oh KJ, Kim G, Kwon JY. Non-invasive prediction of intra-amniotic inflammation in women with preterm labor. Ultrasound Obstet Gynecol 2011; 37: 82-7.

15. Thomakos $N$, Daskalakis $G$, Papapanagiotou A, Papantoniou $N$ Mesogitis S, Antsaklis A. Amniotic fluid interleukin-6 and tumor necrosis factor-alpha at mid-trimester genetic amniocentesis: relationship to intra-amniotic microbial invasion and preterm delivery. Eur J Obstet Gynecol Reprod Biol 2010; 148: 147-51.

16. Lowell DM, Kaplan C, Salafia CM. College of American Pathologists Conference XIX on the examination of the placenta: report of the working group on the definition of structural changes associated with abnormal functions in the maternal-fetal-placental unit in the second and third trimesters. Arch Pathol Lab Med 1991; 115: 647-731.

17. Uzar E, Tamam Y, Evliyaoglu O, Tuzcu A, Beyaz C, Acar A, Aydın B, Tasdemir N. Serum prolidase activity and oxidative status in patients with diabetic neuropathy. Neurol Sci 2011; [Epub ahead of print].

18. Kosecik M, Erel O, Sevinc E, Selek S. Increased oxidative stress in children exposed to passive smoking. Int J Cardiol 2005; 100: 61e4.

19. Aebi H. Catalase in vitro. Methods Enzymol 1984; 105: 121-6.

20. Eckerson HW, Wyte CM, La Du BN. The human serum paraoxonase/arylesterase polymorphism. Am J Hum Genet 1983; 35: 1126-38.

21. Kayabasi H, Sit D, Atay AE, Yilmaz Z, Kadiroglu AK, Yilmaz ME. Parameters of oxidative stress and echocardiographic indexes in patients on dialysis therapy. Ren Fail 2010; 32: 328-34.

22. [Internet] Statistics Calculators. http://www.danielsoper.com/ statcalc3/
23. Fortunato SJ, LaFleur B, Menon R. Collagenase-3 (MMP-13) in fetal membranes and amniotic fluid during pregnancy. Am J Reprod Immunol 2003; 49: 120-5.

24. Biggio JR Jr, Ramsey PS, Cliver SP, Lyon MD, Goldenberg RL, Wenstrom KD. Midtrimester amniotic fluid matrix metalloproteinase-8 (MMP-8) levels above the 90th percentile are a marker for subsequent preterm premature rupture of membranes. Am J Obstet Gynecol 2005; 192: 109-13.

25. Maymon E, Romero R, Pacora P, Gervasi MT, Edwin SS, Gomez R, Seubert DE. Matrilysin (matrix metalloproteinase 7) in parturition, premature rupture of membranes, and intrauterine infection. Am J Obstet Gynecol 2000; 182: 1545-53.

26. Athayde N, Edwin SS, Romero R, Gomez R, Maymon E, Pacora P. A role for matrix metalloproteinase- 9 in spontaneous rupture of the fetal membranes. Am J Obstet Gynecol 1998; 179: 1248-53.

27. Maymon E, Romero R, Pacora P, Gervasi M-T, Bianco K, Ghezzi F, Yoon $\mathrm{BH}$. Evidence for the participation of interstitial collagenase (matrix metalloproteinase-1) in preterm premature rupture of membranes. Am J Obstet Gynecol 2000; 183: 914-920.

28. Liu G, Nakayama K, Awata S, Tang S, Kitaoka N, Manabe M, Kodama H. Prolidase isoenzymes in the rat: their organ distribution, developmental change and specific inhibitors. Pediatr Res 2007; 62: 54-9.

29. Myara I, Myara A, Mangeot M, Fabre M, Charpentier C, Lemonnier A. Plasma prolidase activity: A possible index of collagen catabolism in chronic liver disease. Clin Chem 1984; 30: 211-15.

30. Cechowska-Pasko M, Pałka J, Wojtukiewicz MZ. Enhanced prolidase activity and decreased collagen content in breast cancer tissue. Int J Exp Pathol 2006; 87: 289-96.

31. Gumus S, Yaman H, Ozcan O, Deniz O, Karaman B, Cakir E, Tozkoparan E, Ozkan M, Bilgic H. Serum prolidase activity in patients with pulmonary tuberculosis. Scand J Clin Lab Invest 2011; 71: 467-72.

32. Buhimschi IA, Kramer WB, Buhimschi CS, Thompson LP, Weiner CP. Reduction-oxidation (redox) state regulation of matrix metalloproteinase activity in human fetal membranes. Am J Obstet Gynecol 2000; 182: 458-64.

33. Longini M, Perrone S, Vezzosi P, Marzocchi B, Kenanidis A, Centini G, Rosignoli L, Buonocore G. Association between oxidative stress in pregnancy and preterm premature rupture of membranes. Clin Biochem 2007; 40: 793-7.

34. Osaikhuwuomwan JA, Okpere EE, Okonkwo CA, Ande AB, Idogun ES. Plasma vitamin $C$ levels and risk of preterm prelabour rupture of membranes. Arch Gynecol Obstet 2011; 284: 593-7.

35. Zalud I, Janas S. Risks of third-trimester amniocentesis. J Reprod Med 2008; 53: 45-8. 